\title{
Smaltimento illecito dei rifiuti: possibile fonte di alterazione dello stato di qualità dei suoli
}

\author{
Federica Pasquarelli ${ }^{1}$, Barbara D’ Alessio $^{1}$, and Grazia Senese ${ }^{1}$ \\ ${ }^{1}$ Affiliation not available
}

\begin{abstract}
Il comparto suolo e sottosuolo è uno dei comparti che maggiormente viene approfondito per la redazione degli studi di impatto ambientale. La sua caratterizzazione gioca un ruolo fondamentale nell'ambito della disciplina ambientale, sia per definire lo stato preesistente dell'area oggetto di studio, sia per valutare come l'intervento proposto potrebbe alterare tale condizione.

Nel seguente lavoro si descrivono, dunque, gli strumenti utili per rilevare e studiare le principali caratteristiche delle componenti e dei fattori ambientali e si riporta l'analisi di un caso studio relativo ad una vasta area soggetta al preoccupante fenomeno della combustione dei rifiuti illecitamente abbandonati.

L'approfondimento del caso studio si pone l'obiettivo di illustrare le analisi effettuate e i risultati ottenuti, in termini di classificazione dei terreni in classi di rischio ai fini dell'uso agricolo, per garantire la qualità delle produzioni agroalimentari a tutela della salute umana.
\end{abstract}

\section{Introduzione e quadro normativo di riferimento}

Il comparto suolo e sottosuolo, insieme al comparto idrico e quello atmosferico, riveste un ruolo importante per il corretto sviluppo delle specie viventi ${ }^{1,2,3}$, motivo per il quale una sua approfondita caratterizzazione risulta indispensabile nelle valutazioni di impatto ambientale ${ }^{4,5}$. Il suolo rappresenta lo strato superficiale della crosta terrestre in cui sono presenti ossigeno e sostanze nutritive 
necessarie e utili per la nascita e il sostentamento delle specie vegetali; il sottosuolo rappresenta la porzione di crosta e il complesso di rocce che si trovano al di sotto della superficie del suolo, in cui invece è scarsa la presenza di tali sostanze nutritive.

I principali indicatori, utilizzati per la valutazione dello stato di qualità delle suddette componenti, sono la tessitura del suolo, la permeabilità, il contenuto di sostanza organica e di metalli pesanti, la stabilità, la sismicità e il pH, i quali possono essere desunti da specifiche carte tematiche.

Il quadro normativo di riferimento per lo studio di tale comparto ambientale si sostanzia negli allegati I e II contenuti nelle Norme Tecniche per la redazione degli studi di impatto ambientale e la formulazione del giudizio di compatibilità (Decreto del Presidente del Consiglio dei Ministri 27/12/1988). All'allegato I, che illustra le componenti e i fattori ambientali, suolo e sottosuolo sono intesi sotto il profilo geologico, geomorfologico e pedologico, nel quadro dell'ambiente in esame, ed anche come risorse non rinnovabili; secondo l'allegato II, che definisce gli obiettivi della caratterizzazione e dell'analisi del comparto da esaminare, ogni caratteristica ed ogni fenomeno geologico, geomorfologico e geopedologico vengono esaminati come effetto di varie attività an-

tropiche, il cui risultato è rilevabile al momento dell'osservazione ed è prevedibile per il futuro, sia in assenza che in presenza dell'opera progettata ${ }^{6}$.

Elementi conoscitivi fondamentali per la caratterizzazione del comparto suolo e sottosuolo sono i vincoli territoriali, ovvero idraulico, idrogeologico, sismico e derivante da incendio, i quali possono essere imposti da direttive comunitarie od anche da leggi nazionali o regionali.

\section{Caso studio}

Con il termine Terra dei fuochi si indica un vasto territorio situato tra le province di Napoli e Caserta e comprendente circa 90 comuni, così denominato a causa dei continui incendi di rifiuti illecitamente abbandonati, i quali, se correttamente gestiti, potrebbero rappresentare una risorsa 
anziché un potenziale pericolo ${ }^{7,8,9}$.

Decenni di queste criminose attività, potrebbero aver comportato conseguenze estremamente pericolose, oltre che per la salute umana, anche per le matrici ambientali ${ }^{10}$.

Alla luce di quanto detto, con l'emanazione del Decreto Legge 10 dicembre 2013, n.136, il Governo ha ritenuto necessario che fossero svolte delle indagini tecniche e delle analisi specifiche sui terreni della Regione Campania destinati all'agricoltura al fine di valutare la possibile contaminazione.

Per l'attuazione di quanto previsto in questo decreto, è stato istituito un apposito Gruppo di Lavoro formato da Istituto Superiore per la Protezione e la Ricerca Ambientale (ISPRA), Consiglio per la Ricerca e la sperimentazione e l'Agricoltura (CRA), Istituto Superiore di Sanità (ISS), Agenzia Regionale per la Protezione Ambientale in Campania (ARPAC) e Agenzia per le Erogazioni in Agricoltura (AGEA); tali enti, oltre ad individuare i territori, ricadenti in 57 comuni, da sottoporre urgentemente ad analisi, hanno definito un Modello Scientifico utilizzato per la classificazione dei terreni agricoli, finalizzata a garantire la qualità delle produzioni agroalimentari a tutela della salute umana ( Direttiva Ministeriale 23/12/2013).

Il modello si compone di sette fasi:

1. Individuazione dei siti ed integrazione geografica

2. Individuazione degli inquinanti indice

3. Definizione delle relazioni acqua-suolo-pianta-animale-catena alimentare

4. Costruzione di un indice per l'individuazione delle classi di rischio per la salute umana, animale e di un indice di rischio dei siti di gestione/abbandono dei rifiuti

5. Proposta di classificazione dei terreni ai fini dell'uso agricolo

6. Definizione degli interventi di risanamento

7. Verifica e controllo 
Combinando i dati disponibili sui suoli agricoli con ortofoto multi-temporali, è stato possibile suddividere i diversi siti in esame in 5 livelli di rischio potenziale:

Agli allegati A, B, C e D del Decreto Interministeriale 11 marzo 2014 è riportata la suddivisione dei terreni nelle diverse classi di rischio presunto.

Per i terreni rientranti nella classe di rischio presunto 5, 3 e 2 b sono state effettuate indagini radiometriche superficiali, per valutare i livelli di radioattività dello strato superficiale del suolo, indagini geomagnetometriche, per valutare la possibile presenza di rifiuti interrati, campionamenti di suolo e acque utilizzate per scopi irrigui, limitatamente ai siti aventi pozzi campionabili, campionamento di prodotti agricoli o vegetazione spontanea ed infine accertamenti visivi per valutare la possibile presenza di rifiuti in superficie.

Le stesse indagini sono state effettuate anche per i terreni rientranti nella classe di rischio presunto 4, 2a, 2c, 2d e 1, eccezion fatta per le indagini radiometriche e geomagnetometriche.

Nel loro complesso, i risultati delle indagini effettuate hanno permesso di classificare i terreni in diverse classi di rischio ai fini dell'uso agricolo, in base al verificarsi o meno di particolari condizioni:

\section{A: terreni idonei alle produzioni agroalimentari}

1. concentrazioni inquinanti $<\mathrm{CSC}$ (o VF) o $>$ VF ma non biodisponibili

2. prodotti agroalimentari o vegetali spontanei conformi ai limiti normativi

3. indagini geomagnetometriche con esito negativo

4. assenza di rifiuti in superficie

B: terreni con limitazione a determinate produzioni agroalimentari in determinate condizioni

1. concentrazione inquinanti $>\mathrm{CSC}$ (o VF); sono presenti $\mathrm{PCDD} / \mathrm{PCDF}$ in concentrazioni $<\mathrm{a}$ $6 \mathrm{ng} / \mathrm{kg}$ ss, ma comunque superiori ai livelli presenti nei terreni di classe A 
2. prodotti agroalimentari o vegetali spontanei conformi ai limiti normativi ma con concentrazione degli inquinanti di poco inferiori al limite; vegetazione spontanea non conforme ai limiti

3. indagini geomagnetometriche con esito negativo

C: terreni idonei alle produzioni non agroalimentari

1. terreni negativi alle indagini geomagnetometriche, ma nei quali si rilevano processi di biodisponibilità degli inquinanti

D: terreni con divieto di produzioni agroalimentari e silvo pastorali

1. presenza di uno o più inquinanti in concentrazioni molto superiori alle CSC

2. prodotti agroalimentari o vegetali spontanei non conformi ai limiti normativi

3. presenza di rifiuti interrati già negli strati superficiali del suolo

4. indagini geomagnetometriche con esito positivo

In particolare, per quanto riguarda i siti con livello di rischio presunto 4 e 5 , sono classificati nella classe A circa il $36 \%$, nella classe D circa il $37 \%$ e il restante $27 \%$ nella classe B:

Per quanto riguarda invece i siti con livello di rischio presunto 3, sono classificati nella classe A circa l' $85 \%$, nella classe D circa 1'11\% e il restante $4 \%$ nella classe B:

In ultimo, per quanto riguarda i siti con livello di rischio presunto 2 a e i terreni limitrofi a quelli con livello di rischio presunto 4 e 5 (siti con livello di rischio presunto 4 e 5 estensione), sono classificati nella classe A circa il $69 \%$, nella classe D circa il $6 \%$ e il restante $25 \%$ nella classe B:

Per una più semplice fruizione dei risultati ottenuti, questi ultimi sono stati implementati in ambiente GIS, cosicché fosse possibile consultare e interrogare delle mappe interattive riportanti i principali riferimenti catastali e le informazioni ai fini dell'uso agricolo di tutti i terreni oggetto di 
indagine.

Infine, è stato rilevato che 8 comuni presentano terreni di classe $\mathrm{D}$, per i quali recentemente è stato messo a punto un regolamento concernente gli interventi di bonifica e il ripristino ambientale delle aree destinate alla produzione agricola e all'allevamento, ai sensi dell'articolo 241 del Decreto Legislativo 3 aprile 2006, n.152 (Decreto 01/03/2019,n.46).

\section{Conclusioni}

I risultati emersi hanno mostrato l'assenza di valori anomali di radioattività dello strato superficiale del suolo, mentre sono state riscontrate positività in 28 terreni in seguito alle indagini geomagnetometriche.

Dalle analisi chimico-fisiche e microbiologiche è emerso che nessuno dei prodotti agricoli per l'alimentazione umana è risultato non conforme ai limiti normativi, tuttavia in 5 campioni di vegetazione spontanea sono state riscontrate delle non conformità.

Gli inquinanti riscontrati con maggiore frequenza sono stati le diossine, gli idrocarburi policiclici aromatici ed alcuni metalli pesanti; dalle analisi per la conoscenza della biodisponibilità dei metalli sono emersi valori di mobilità molto bassi, a causa del pH sub-alcalino e dell' elevato contenuto in argilla, calcare e sostanza organica dei suoli.

Complessivamente, dunque, dei quasi 240 ettari di superficie agricola analizzata il $67 \%$ risulta idonea alle produzioni agroalimentari, il $13 \%$ presenta il divieto di produzioni agroalimentari e silvo pastorali, mentre il $20 \%$ rientra nei terreni con limitazioni circa determinate produzioni agroalimentari in determinate condizioni. 


\section{References}

1.Murena, A. et al.. Water-Energy Nexus: Evaluation of the Environmental Impact on the National and International Scenarios. in Frontiers in Water-Energy-Nexus-Nature-Based Solutions Advanced Technologies and Best Practices for Environmental Sustainability 33-35 (Springer International Publishing, 2019). doi:10.1007/978-3-030-13068-8.

2.Hasan, S. W., Liu, H., Naddeo, V., Puig, S. \& Yip, N. Y. Environmental Technologies for the Sustainable Development of the Water and Energy Sectors. (IWA Publishing, 2020). doi:10.2166/9781789062328.

3.Naddeo, V., Balakrishnan, M. \& Choo, K. H. Frontiers in Water-Energy-Nexus. (Springer International Publishing, 2020).

4.Naddeo, V., Belgiorno, V., Zarra, T. \& Scannapieco, D. Dynamic and embedded evaluation procedure for strategic environmental assessment. Land Use Policy 31, 605-612 (2013).

5.Nesticò, A., Elia, C. \& Naddeo, V. Sustainability of urban regeneration projects: Novel selection model based on analytic network process and zero-one goal programming. Land Use Policy 99 , 104831 (2020).

6.Scannapieco, D., Naddeo, V. \& Belgiorno, V. Sustainable power plants: A support tool for the analysis of alternatives. Land Use Policy 36, 478-484 (2014). 
7.Naddeo, V. \& Korshin, G. Water energy and waste: The great European deal for the environment. Science of The Total Environment 764, 142911 (2021).

8.Cesaro, A., Naddeo, V., Amodio, V. \& Belgiorno, V. Enhanced biogas production from anaerobic codigestion of solid waste by sonolysis. Ultrasonics Sonochemistry 19, 596-600 (2012).

9.Cesaro, A., Belgiorno, V. \& Naddeo, V. A comparative technology assessment of the anaerobic digestion of an organic fraction of municipal solid waste. The Sustainable World - Transactions on Ecology and the Environment vol. 83 145-156, 2011.

10.Naddeo, V., Rizzo, L. \& Belgiorno, V. Water, wastewater and soil treatment by advanced oxidation processes. (ASTER, 2011). 


\section{Figure Captions}

Figure 1. Suolo (fone: unsplash.com)

Figure 2. Discarica di Caivano (NA) (fonte: Internazionale.it)

Figure 3. Livelli di rischio presunto

Figure 4. Campionamento di suolo eseguito da un operatore ARPAC (fonte: ilfattoquotidiano.it)

Figure 5. Classi di rischio ai fini dell'uso agricolo

Figure 6. Valutazione terreni delle classi di rischio presunto 4 e 5

Figure 7. Valutazione terreni della classe di rischio presunto 3

Figure 8. Valutazione terreni delle classi di rischio 2a, 4 e 5 estensione 


\section{Figures}

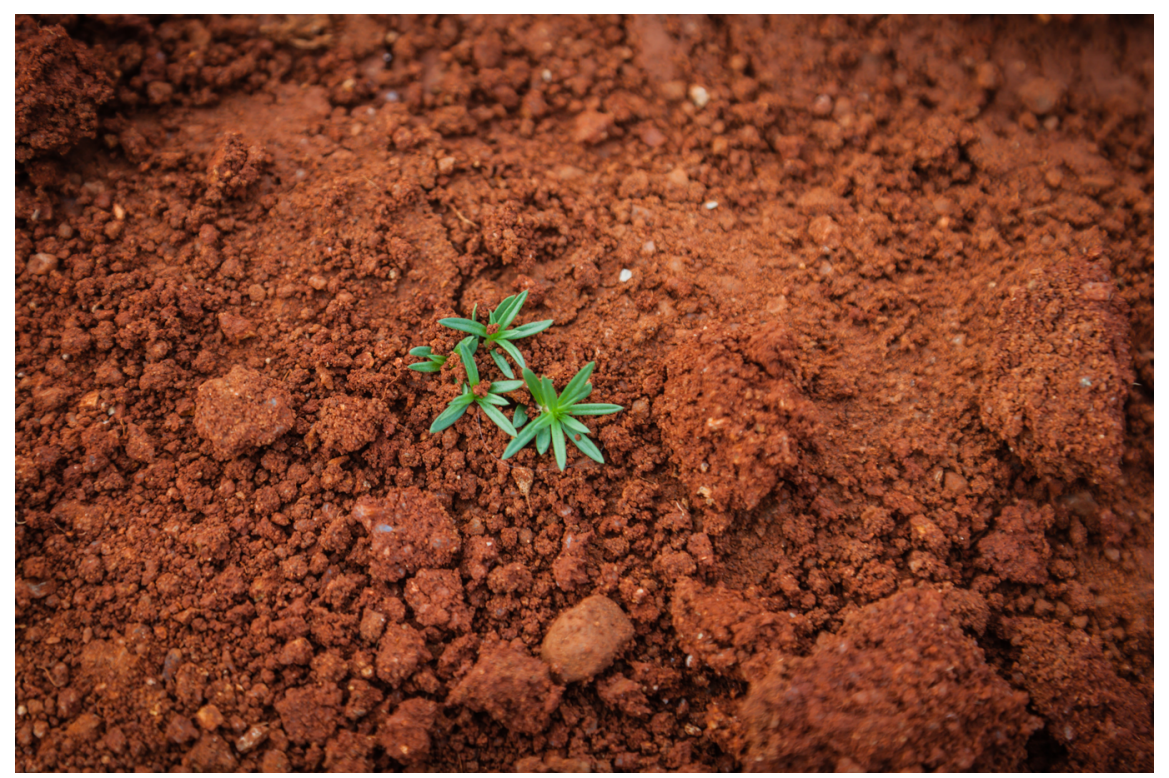

Figure 1: Suolo (fone: unsplash.com)

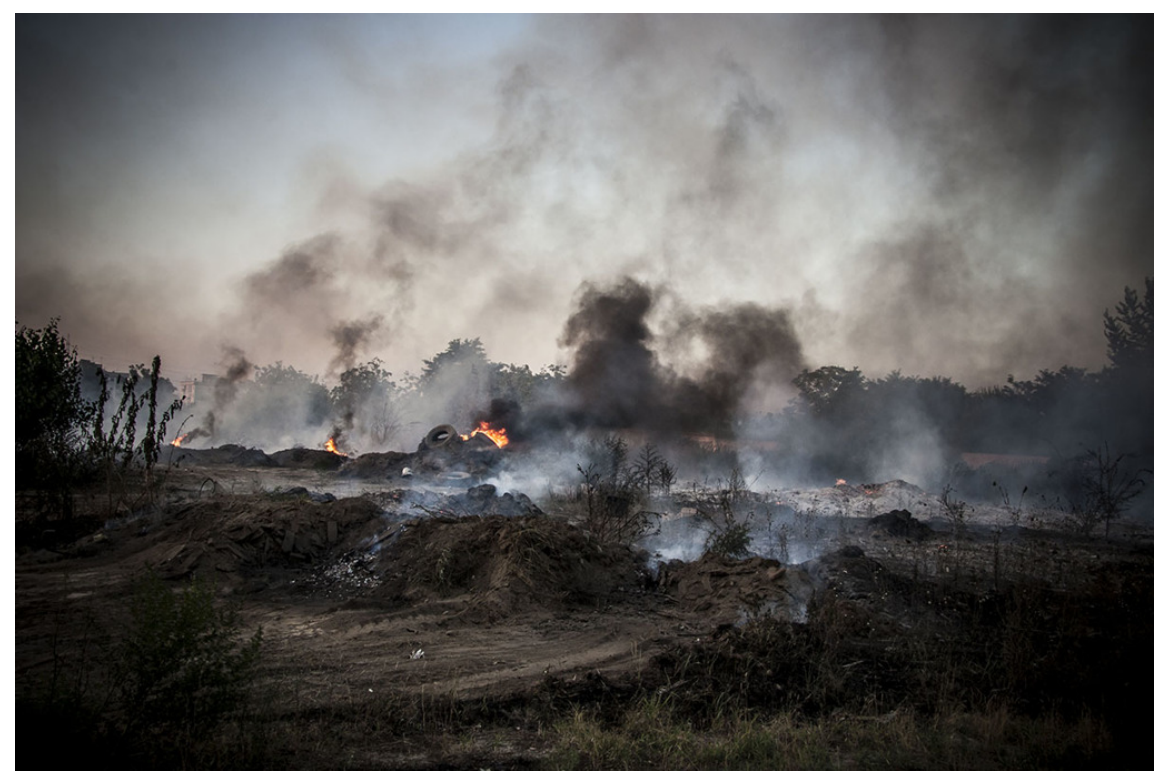

Figure 2: Discarica di Caivano (NA) (fonte: Internazionale.it) 


\begin{tabular}{|c|l|}
\hline $\begin{array}{c}\text { Livelli di rischio } \\
\text { presunto }\end{array}$ & \multicolumn{1}{|c|}{ Caratteristiche del sito } \\
\hline 5 & $\begin{array}{l}\text { Valore inquinanti > 10 CSC (o VF) e corrispondenza con siti a rischio da } \\
\text { analisi foto aeree }\end{array}$ \\
\hline 4 & Valore inquinante > 10 CSC (o VF) \\
\hline 3 & $\begin{array}{l}\text { Valore inquinanti = 2-10 CSC (o VF) e corrispondenza (entro 10 m) con } \\
\text { siti a rischio da analisi foto aeree }\end{array}$ \\
\hline $2 \mathrm{a}$ & Valore Inquinanti = 2-10 CSC (o VF) \\
\hline $2 \mathrm{~b}$ & Siti a rischio da analisi foto aeree \\
\hline $2 \mathrm{c}$ & $\begin{array}{l}\text { Aree agricole delle aree vaste Lo Uttaro, Bortolotto-Sogeri e Masseria del } \\
\text { Pozzo, aree agricole del PRB }\end{array}$ \\
\hline $2 \mathrm{~d}$ & $\begin{array}{l}\text { Aree agricole circostanti impianti di smaltimento rifiuti, aree industriali, } \\
\text { grandi arterie di traffico veicolare e aste del sistema dei Regi Lagni, aree } \\
\text { incendi di grande rilevanza, siti a rischio da analisi foto aeree }\end{array}$ \\
\hline 1 & Valore inquinanti < 2 CSC (o VF) \\
\hline
\end{tabular}

Figure 3: Livelli di rischio presunto

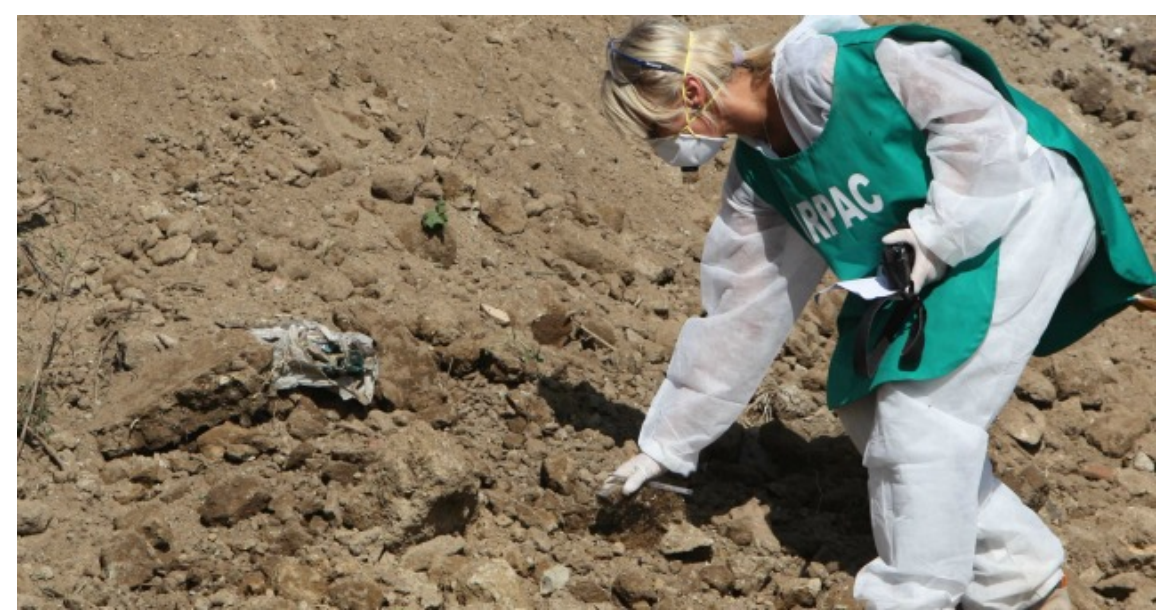

Figure 4: Campionamento di suolo eseguito da un operatore ARPAC (fonte: ilfattoquotidiano.it) 


\begin{tabular}{|c|l|}
\hline $\begin{array}{c}\text { Classi di rischio ai fini } \\
\text { dell' uso agricolo }\end{array}$ & \multicolumn{1}{|c|}{ Stato } \\
\hline A & Si verificano tutte le condizioni \\
\hline B & $\begin{array}{l}\text { Si verifica la condizione 3 con una delle } \\
\text { condizioni 1 e/o una delle condizioni 2 }\end{array}$ \\
\hline C & Si verifica la condizione 1 \\
\hline D & Si verifica almeno una delle condizioni \\
\hline
\end{tabular}

Figure 5: Classi di rischio ai fini dell'uso agricolo

\begin{tabular}{|c|c|}
\hline Status del sito & Superficie (ha) \\
\hline Classe A & 15,57 \\
\hline Classe B & 11,60 \\
\hline Classe C & 0,00 \\
\hline Classe D & 15,78 \\
\hline Totale & 42,95 \\
\hline
\end{tabular}

Figure 6: Valutazione terreni delle classi di rischio presunto 4 e 5

\begin{tabular}{|c|c|}
\hline Status del sito & Superficie (ha) \\
\hline Classe A & 41,91 \\
\hline Classe B & 2,13 \\
\hline Classe C & 0,00 \\
\hline Classe D & 5,33 \\
\hline Totale & 49,37 \\
\hline
\end{tabular}

Figure 7: Valutazione terreni della classe di rischio presunto 3 


\begin{tabular}{|c|c|}
\hline Status del sito & Superficie (ha) \\
\hline Classe A & 101,02 \\
\hline Classe B & 35,27 \\
\hline Classe C & 0,00 \\
\hline Classe D & 8,97 \\
\hline Totale & 145,26 \\
\hline
\end{tabular}

Figure 8: Valutazione terreni delle classi di rischio 2a, 4 e 5 estensione 